\title{
An art-based IS for improving room-climate
}

\author{
Paul Rigger and Felix Wortmann \\ University of St. Gallen, Dufourstrasse 40a, 9000 St. Gallen, Switzerland \\ \{paul.rigger, felix.wortmann\}@unisg.ch
}

\begin{abstract}
Indoor room-climate influences our everyday life on many levels. High room temperature decreases our productivity and our ability to concentrate. Moreover, many medical conditions can be traced back to bad indoor room-climate. In this paper we present an information system (IS) for roomclimate monitoring. Our system is an informative art system being equipped with standard room climate sensors for temperature, humidity and carbon dioxide. In contrast to conventional monitoring systems a display embedded in a standard art frame presents the current room climate on the basis of classical art. Changing room conditions are reflected in the painting in real-time. By implementing the presented IS we pursue a research avenue which is dedicated to a more fundamental research questions: "Are hedonic, art-based IS superior to utilitarian, non-art-based IS in respect to usage and impact?
\end{abstract}

Keywords. Human-computer interaction, ambient displays, art information systems, pervasive computing

\section{$1 \quad$ Introduction}

In 1983 the World Health Organization defined the sick building syndrome, describing the influence of poor indoor climate on human beings, causing discomforts and health risks. Symptoms include negative effects on eyes, nose, throat and lower airways, skin reactions, non specific hypersensitivity, mental fatigue, headache, nausea, and dizziness among people staying in respective buildings [1].

In contrast to the latest generation of professional buildings, older professional buildings and residential homes are rarely equipped with an automatic ventilation system and fresh air is usually provided through opened windows. However, sensing room climate can be very hard for humans. While we have a good sense for temperature, humidity levels are only recognized with a delay of hours, e.g. on the basis of a dry throat. Even worse, carbon dioxide levels cannot be sensed directly at all. Information technology can be a remedy to these challenges. Though, engaging people tracking their room climate on the basis of sensor systems is far from being easy. While reading the values on a standard gauge is not very appealing, starting up one of the latest room climate smartphone apps on a regular basis is cumbersome. Furthermore, providing enough help to interpret room climate data is challenging.

To address the depicted challenges we propose an art-based IS. Thereby, we pursue a broader research avenue which is dedicated to a fundamental research questions: "Are 
hedonic, art-based IS superior to utilitarian, non-art-based IS in respect to usage and impact?

\section{Design of the Artifact}

\subsection{Problem Statement}

Having analyzed and tested multiple of the currently available solutions in real life, from standard gauges[2] to more sophisticated internet-enabled sensors [3], the following key problems can be identified [4]:

- Complex user interfaces and non-self-explanatory presentation of data: Displaying blunt data on a gauge or a digital display requires a lot of prior user knowledge to be effective. Data has to be interpreted and compared against known target values. While interpretation of temperature can be performed by an average user, e.g. interpreting $\mathrm{CO} 2$ values in parts per million (ppm) can indeed be very challenging.

- Lack of systematic engagement: Room climate is specific for each individual room and has to be measured continuously when the user is present. Furthermore, the room climate IS should specifically engage the user in case of poor room climate conditions. Standard room climate systems tend to keep the level of engagement constant, thereby losing the users intention already during periods of good room climate.

- Long-term usage challenges: As discussed, current systems lack self-explanatory user interfaces and do not engage the user when the room climate conditions worsen. This ultimately challenges their long-term usage and impact. However, there is a new generation of internet-enabled system using smart phone apps or other mobile front-end devices to display information. While these systems overcome some of the discussed challenges they bring their own set of issues. Starting up an app is cumbersome compared to an always visible measurement device. Push notifications can be used as a remedy to inform the user even if she does not start the app. However, these notifications are often perceived as intrusive and disturbing, especially when not being in the corresponding room currently having bad conditions.

\subsection{Requirement Analysis}

Preliminary research in the context of our work can be found under terms such as "ambient information systems" [5],"informative art systems" [6], or "peripheral displays". Various articles derive design principles for successful ambient information systems [6-10]. Building upon this body of knowledge we derive four key design requirements to develop our prototype:

- Connect to people emotionally: We want to go beyond designing a system that builds upon rationality and cognitive thinking, i.e. leverage emotions and psychological incentives [9]. 
- Draw attention only when necessary: We want to build a monitoring device that is unobtrusive and remain so unless attention is really necessary. The device should integrate seamlessly into the surroundings [11].

- Provide choice: The design of the artifact is supposed to foster usage and post acceptance. Therefore, we want to leverage the advantages of personalization [12] and allow the user to customize the solution to a specific taste [8].

- Learn from the past: Apart from having the current room climate displayed on spot with the display, a smartphone application allows to analyze the data in more depth whenever a user wants. To enable rich feedback of the room climate this application also allows a review of historical data.

\subsection{Design and features of the artifact}

The goal of this research is to build a room-climate monitoring system that fosters usage and post acceptance by integrating an innovative user interface, descriptive and injunctive feedback [13] and recommendations for improvements. We do so by implementing a hedonic user-interface by incorporating art.

Therefore we build a standard wooden art frame that is equipped with standard room climate sensors, and internet-capable minicomputer and a LCD display to illustrate art. In contrary to a standard framed picture, the displayed art gets modified in real time according to the continuously measured room climate.

Our first prototype "Quantified-Art" shows Marilyn Monroe and manipulates her face in case of room climate changes. As pointed out by [8] the idea behind using a face is that it is easily recognized including changes in the appearance. More specifically, low temperature is presented by coloring the lips of Marilyn Monroe blue. Likewise high temperatures, exceeding a predefined threshold, transform the lips neon yellow for Marilyn Monroe. The intensity of the lips color reflects the extent that the threshold is being exceed/fallen below. The room's humidity level is presented by modification of the skin. Values below a threshold are depicted as a dry skin with cracks. Exceeding the optimal humidity value forms droplets on the skin. Likewise, the indoor carbon dioxide level is presented by a modification of the skin. The level of exceedance of each measurement is presented by the intensity of the modification. The modification is done in real-time by software deployed on the minicomputer.

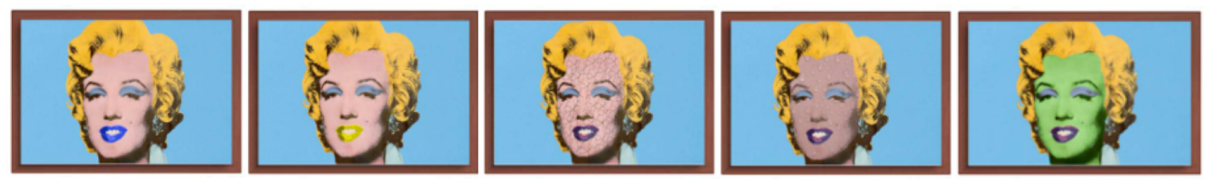

Fig. 1. Modifications according to room-climate conditions (from left: 1. Low temperature, high temperature, low humidity, high humidity, high carbon dioxide level) 
The Quantified-Art display was developed using a 23-inch TFT-LCD panel. Having a brightness of $250 \mathrm{~cd} / \mathrm{m}^{2}$ and viewing angles of $89^{\circ}$ in each direction the displays are not recognized as displays but as a normal print of art. A wooden frame hides the metallic edges of the panel and provides enough space in the back of the display to house the sensors, the computer and electrical power supply. The Raspberry Pi [14], a Linux based small computer, in the back of the display was used to interact with the sensor and display the art using the built in graphic chip. The Raspberry Pi provides 16 GPIO (general purpose input output) and SPI, I2C as well as an AURT interface to connect sensors. Sensing room climate levels we used the K33 OEM [15] module from Sense Air measuring carbon dioxide concentration with a no dispersive infrared sensor. Communicating with the Raspberry Pi the sensor provides a UART interface.

Beside the Quantified-Art display we provide the user with a web portal to change settings. This way the display itself stays a true plug and play product without a complicated user interface. After login with the corresponding Quantified-Art identification the user can change the displayed art as well as adapt the behavior of the art corresponding to the measured room climate. Apart from having the current room climate displayed on spot with the quantified-art display, a smartphone application allows the user to check the room climate on the go

In order to provide the described functionalities, we need a comprehensive architecture. We use a standard SQL database (PostgreSQL) [16] to store historical data, handle user administration and provide threshold data. In addition, the database is linked to a second database where all the art content is available for download. All front-end devices communicate with the backend over a middleware written in NodeJS [17] and deployed on the server. The middleware allows posting new values or users on the backend without accessing the database itself. The same applies to getting data from the server. The devices request specific data (e.g. temperature over the last 24 hours) and the middleware provides it from the backend.

\section{Evaluation of the Artifact and Further Research}

During a pre-evaluation phase the prototype is currently demonstrated in a real world setup and potential users evaluate the artifact via an item-based questionnaire. In a first step, we try to understand the influence of our artifact on motivation as a driver for usage. Based on $[18,19]$ there are different motivations driving human action. Fun and enjoyment are a fundamental source of intrinsic motivation. Furthermore, usefulness and willingness to learn are a second source of so-called internalized extrinsic motivation. This first stage of testing allows the evaluation of the effectiveness of the prototype building upon the work of [20].

In a longitudinal field study the prototypes will be tested in 50 classrooms of a secondary school. The logging of the actual room climate allows A/B testing for the long-term impact of the proposed system compared to classrooms with standard or without any room-climate monitoring system. In addition to quantitative log data, the qualitative feedback of 800 students and teaching stuff will be collected. 
By evaluation and redesigning the proposed prototype we intend to derive a design theory for art-based IS. Besides room climate data further research could expand the focus to other domains. Indeed, we are currently discussing with leading diabetic health care researches to use an art-based IS to monitor and improve blood sugar levels of diabetic patients.

\section{References}

1. Skov, P., Valbjorn, O., Pedersen, B. V: Influence of Indoor Climate on the Sick Building Syndrome in an Office Environment. The Danish Indoor Climate Study Group. Scandinavian Journal of Work, Environment \& Health. 16, 363-371 (1990)

2. Climatemeter Temperature/Humidity - Lufft,

http://www.lufft.com/en/products/temperaturehumidity/climatemeter-511099 (accessed February $28^{\text {th }}, 2014$ )

3. The Netatmo Weather Station, http://www.netatmo.com/en-US/product (accessed February $\left.28^{\text {th }}, 2014\right)$

4. blinded for review

5. Pousman, Z., Stasko, J.: A Taxonomy of Ambient Information Systems. Proceedings of the working conference on advanced visual interfaces - AVI '06. p. 67. ACM Press, New York, New York, USA (2006)

6. Redström, J., Skog, T., Hallnäs, L.: Informative Art. Proceedings of DARE 2000 on Designing augmented reality environments - DARE '00. pp. 103-114. ACM Press, New York, New York, USA (2000)

7. Streitz, N.N.A., Rocker, C., Prante, T., van Alphen, D., Stenzel, R., Magerkurth, C.: Designing Smart Artifacts for Smart Environments. Computer. 38, 41-49 (2005)

8. Ferscha, A.: A Matter of Taste. Ambient Intelligence. 287-304 (2007)

9. Nakajima, T., Lehdonvirta, V.: Designing Motivation Using Persuasive Ambient Mirrors. Personal and Ubiquitous Computing. 17, 107-126 (2011)

10. Matthews, T., Forlizzi, J., Rohrbach, S.: Designing Glanceable Peripheral Displays. UC Berkeley. (2005)

11. Hallnäs, L., Redström, J.: Slow Technology - Designing for Reflection. Personal and Ubiquitous Computing. 5, 201-212 (2001)

12. Kulkarni, A.: Design Principles of a Reactive Behavioral System for the Intelligent Room. Bitstream: The MIT Journal of EECS Student Research. 1-6 (2002)

13. Loock, C., Staake, T., Thiesse, F.: Motivating Energy-Efficient Behavior with Grenn IS: An Investigation of Goal Setting and the Role of Defaults. MIS Quaterly. 37, 1313-1332 (2013)

14. Raspberry Pi I An ARM GNU/Linux box, http://www.raspberrypi.org (accessed February $\left.28^{\text {th }}, 2014\right)$

15. CO2Meter.com CO2, Temperature, Humidty, Environmental Data Logger, http://www.co2meter.com/collections/co2-sensors/products/k33-environmental-logger-co2sensor (accessed February $28^{\text {th }}, 2014$ )

16. PostgresSQL, http://www.postgresql.org (accessed February $28^{\text {th }}, 2014$ )

17. node.js, http://nodejs.org (accessed February $28^{\text {th }}, 2014$ )

18. Deci, E., Ryan, R.: The "What" and "Why" of Goal Pursuits: Human Needs and the SelfDetermination of Behavior. Psychological inquiry. 11, 227-268 (2000)

19. Ryan, R.M., Deci, E.L.: Self-determination theory and the facilitation of intrinsic motivation, social development, and well-being. The American psychologist. 55, 68-78 (2000)

20. Heijden, H. Van der: User Acceptance of Hedonic Information Systems. MIS quarterly. 28, 695-704 (2004) 